\title{
An Isogeometric Error Estimate for Transport Equation in 2D
}

\author{
Aurélien Goudjo1, Uriel Aguemon² \\ ${ }^{1}$ FAculté des Sciences et Techniques (FAST), Université d'Abomey-Calavi (UAC), Abomey-Calavi, Benin \\ ${ }^{2}$ Institut de Mathématiques et de Sciences Physiques (IMSP), Université d'Abomey-Calavi (UAC), Abomey-Calavi, Benin \\ Email: aguemonuriel@yahoo.fr
}

How to cite this paper: Goudjo, A. and Aguemon, U. (2019) An Isogeometric Error Estimate for Transport Equation in 2D. Advances in Pure Mathematics, 9, 777-793. https://doi.org/10.4236/apm.2019.99037

Received: September 7, 2019

Accepted: September 15, 2019

Published: September 18, 2019

Copyright (c) 2019 by author(s) and Scientific Research Publishing Inc. This work is licensed under the Creative Commons Attribution International License (CC BY 4.0).

http://creativecommons.org/licenses/by/4.0/

\begin{abstract}
In this paper, an isogeometric error estimate for transport equation is obtained in $2 \mathrm{D}$ to prove the convergence of isogeometric method. The result that we have obtained, generalizes Ern result, got in finite elements method. For the time discretization, the two stage Heun scheme is used to prove this result. For a polynomial of degree $k \geq 1$, the order of convergence in space is 2 and in time is $k+\frac{1}{2}$.

\section{Keywords}

Error Estimate, Isogeometric Method, The Two Stage Heun Scheme, Transport Equation
\end{abstract}

\section{Introduction}

Some phenomena of the daily life such as particles transport in an electric field, the signal transport along a wire, evolution of cars on a road [1], and evolution of a pollutant in a narrow channel [2] are modelled by a transport equation. Study of numerical methods for solving this equation is very important to describe, to predict and to control these phenomena.

Isogeometric Analysis has been introduced by Thomas Hughes, Austin Cottrell and Yuri Bazilevs in 2005 [3].

The objectives of Isogeometric Analysis are to generalize and improve upon Finite Element Analysis (FEA) in the following ways:

1) To provide more accurate modeling of complex geometries and to exactly represent common engineering shapes such as circles, cylinders, spheres, ellipsoids, etc.

2) To fix exact geometries at the coarsest level of discretization and eliminate 
geometrical errors.

3) To vastly simplify mesh refinement of complex industrial geometries by eliminating the necessity to communicate with the $C A D$ (Computer Aided Design) description of geometry.

4) To provide refinement procedures, including classical $h$ - and $p$-refinements analogues, and to develop a new refinement procedure called $k$-refinement [4].

The idea of Isogeometric Analysis is to build a geometry model and, rather than develop a finite element model approximating the geometry, directly use the functions describing the geometry in analysis [5] [6]. These functions are B-splines.

Isogeometric Analysis is approached, using continuous or discontinuous Galerkin method. In the context of space semidiscretization by discontinuous Galerkin methods, explicit $R K$ schemes are used to approximate in time systems of ordinary differential equations. These schemes have been developed by Cockburn and Shu [7], Cockburn, Lin, and Shu [8], and Cockburn, Hou, and Shu [9] and applied to a wide range of engineering problems [10]. They have been used by Alexandre Ern et al. [11] [12], for linear conservation laws using Discontinuous Galerkin Method to prove a convergence result [12]. Authors did a space semidiscretization using the upwind $D G$ method. Besides, others tools are fundamental to get this convergence result:

1) Error equation.

2) An energy identity obtained from error equations.

3) A stability estimate using Gronwall lemma, Young inequality and inverse and trace inequalities for finite elements method.

In the literature, there exist many numerical methods to solve transport equation [13] [14]. To our best knowledge, there is no error estimate for transport equation using isogeometric method. In our work, we give such an estimate to generalize results obtained by Alexandre Ern et al. [11] [12] in finite elements. In the framework of this dissertation, we want to prove a convergence result using isogeometric method. Among others, unlike finite elements, as far as the space semidiscretization is concerned, we have:

1) Constructed a parametrization of the physical domain, indispensable to describe this domain.

2) Constructed a parametric mesh making a tensor product of knot vectors.

3) Introduced the discrete space on the physical domain, using our parametrization.

Moreover, instead of using inverse and trace inequalities for finite elements method, we will use isogeometric inverse and trace inequalities to obtain our convergence result. As far as the discretization in time is concerned, the explicit two stage Heun scheme is used. Now, we consider the following model:

$$
\left\{\begin{array}{l}
\partial_{t} u(x, t)+\nabla \cdot(\beta(x) u(x, t))=0, x \in \Omega \subset \mathbb{R}^{2}, t \in\left[0 ; t_{f}\right] \\
u(., t=0)=u_{0} \\
u=0 \text { in } \partial \Omega^{-} \times\left[0 ; t_{f}\right]
\end{array}\right.
$$


where $\Omega$ is a bounded open set in $\mathbb{R}^{2}, u: \Omega \times\left[0 ; t_{f}\right] \mapsto \mathbf{R}^{2}$ is a scalar-valued function representing the unknown, $t_{f}$ is a finite time,

$\partial \Omega^{-}=\{x \in \partial \Omega, \beta(x) \cdot n(x)<0\}, n$ is the unit outward normal to the domain boundary, $\beta$ is the advective velocity, $\beta \in\left[L^{\infty}(\Omega)\right]^{2}, \nabla \cdot \beta \in L^{\infty}(\Omega)$ and $u_{0}$ is the initial datum.

Let us introduce some notations and assumptions:

- Assume $\beta$ is a Lipschitz continuous functions i.e.

$$
\exists L_{\beta}, \forall(x, y) \in \Omega^{2},\|\beta(x)-\beta(y)\| \leq L_{\beta}\|x-y\| .
$$

where $\|x-y\|$ denotes the Euclidean norm of $(x-y)$ in $\mathbf{R}^{2}$.

- We set $\tau_{c}:=\frac{1}{\max \left\{\left\|\beta^{\prime}\right\|_{L^{\infty}(\Omega)} ; L_{\beta}\right\}}$ and $\beta_{c}:=\|\beta\|_{\left[L^{\infty}(\Omega)\right]^{2}}$.

- We set $\tau_{\star}:=\min \left(t_{f}, \tau_{c}\right)$.

- Assume $h \leq \beta_{c} \tau_{\star}$.

- $\forall x \in \mathbb{R}, x^{\ominus}:=\frac{1}{2}(|x|-x)$ and $x^{\oplus}:=\frac{1}{2}(|x|+x)$.

- Let $l \in \mathbb{N}$, we consider the space

$$
C^{l}(V):=C^{l}\left(\left[0, t_{f}\right], V\right),
$$

where $V$ is a Hilbert space and equipped with the scalar product defined by:

$$
(u, s)_{V}=(u, s)_{L^{2}(\Omega)}+(\nabla \cdot(\beta u), \nabla \cdot(\beta s))_{L^{2}(\Omega)}, \forall(u, s) \in V^{2} .
$$

The associated norm is:

$$
\|u\|_{V}^{2}=\|u\|_{L^{2}(\Omega)}^{2}+\|\nabla \cdot(\beta u)\|_{L^{2}(\Omega)}^{2}, \forall u \in V . \quad[11] \quad \text { (page 39) }
$$

This paper is organized as follows. In the first section, we will describe univariate B-splines. In the second one, we will describe bivariate B-splines and geometry of the physical domain. In the third one, we present main results of this work. In the fourth one, we will state inverse and isogeometric inequalities. In the fifth one, we will talk about the functional setting and space semidiscretization. In the sixth one, we will look into the explicit two stage Heun scheme analysis.

\section{Univariate B-Splines}

Definition 1. Let $x_{1} \leq x_{2} \leq \cdots \leq x_{m}$ be an increasing sequence of reals, $B$-splines functions of degree $k$ are defined by Cox-de Boor-Mansfield recursion formula [15]:

$$
\begin{gathered}
\left\{\begin{array}{l}
\text { For } 1 \leq i \leq m-1 \\
N_{i, 0}(t)=1 \quad \text { if } t \in\left[x_{i}, x_{i+1}[\right. \\
N_{i, 0}(t)=0 \quad \text { otherwise }
\end{array}\right. \\
\left\{\begin{array}{l}
\text { For } k \geq 1 \text { and } 1 \leq i \leq m-k-1 \\
N_{i, k}(t)=\frac{t-x_{i}}{x_{i+k}-x_{i}} N_{i, k-1}(t)+\frac{x_{i+k+1}-t}{x_{i+k+1}-x_{i+1}} N_{i+1, k-1}(t),
\end{array}\right.
\end{gathered}
$$


with the convention $\frac{x}{0}:=0$ for all real number $x$.

The set $\left(x_{i}\right)_{i=1}^{m}(1 \leq i \leq m)$ is called knots vector.

Now, we want to look into bivariate B-splines, obtained from univariate B-splines.

\section{Bivariate B-Splines and Geometry of the Physical Domain}

The definition of bivariate B-splines follows easily through a tensor-product construction. Let us focus on the two-dimensional case. Notably, let us consider the unit square $\hat{\Omega}=[0 ; 1]^{2} \subset \mathbb{R}^{2}$. Mimicking the one-dimensional case, given integers $p_{l}$ and $n_{l}$ for $l=1,2$. Let us introduce open knot vectors:

$$
E_{l}=\left\{\xi_{1, l}, \cdots, \xi_{n_{l}+p_{l}+2, l}\right\}
$$

and the associated vectors without repetitions for each direction $I$

$$
\zeta_{l}=\left\{\zeta_{1, l}, \cdots, \zeta_{m_{l}, l}\right\}
$$

There is a parametric cartesian mesh $Q_{h}$ associated with these knot vectors partitioning the parametric domain $\hat{\Omega}$ into a rectangular grid. So, we have:

$$
Q_{h}=\left\{Q=\otimes_{l=1,2}\left(\zeta_{i_{l}, l}, \zeta_{i_{l+1}, d}\right), 1 \leq i_{l} \leq m_{l}-1\right\} \quad[16]
$$

For each element $Q \in Q_{h}$, we associate a parametric mesh size $h_{Q}=h_{Q, \max }$ where $h_{Q, \max }$ denotes the length of the largest edge of $Q$. Also, for each element, we define a shape regularity constant as in [16]:

$$
\lambda_{Q}=\frac{h_{Q}}{h_{Q, \min }}
$$

where $h_{Q, \min }$ denotes the length of the smallest edge of $Q$.

We associate with each knot vector $E_{l},(l=1,2)$ univariate B-spline basis functions $N_{i, p_{l}}$ of degree $p_{l}$ for $i=1, \cdots, n_{l}$.

On the mesh $Q_{h}$, we define the tensor-product B-spline basis functions as in [16] by:

$$
\begin{aligned}
& N_{(i, j),\left(p_{1}, p_{2}\right)}=N_{i, p_{1}} \otimes N_{j, p_{2}}, i=1, \cdots, n_{1}, j=1, \cdots, n_{2} . \\
& N_{(i, j),\left(p_{1}, p_{2}\right)}=N_{i, p_{1}} N_{j, p_{2}}, i=1, \cdots, n_{1}, j=1, \cdots, n_{2} .
\end{aligned}
$$

The span of these functions form the space of two-dimensional splines over $\hat{\Omega}$, denoted by:

$$
S_{h}=\operatorname{span}\left\{N_{(i, j),\left(p_{1}, p_{2}\right)}\right\}_{i=1, j=1}^{n_{1}, n_{2}}
$$

The physical domain $\Omega$ is defined through a geometrical mapping:

$$
F=\sum_{i=1}^{n_{1}} \sum_{j=1}^{n_{2}} P_{i j} N_{i, n_{1}} N_{j, n_{2}} \quad[16]
$$

where $P_{i j} \in \mathbb{R}^{2}$ are the so-called control points. $F$ is a parametrization of the physical domain $\Omega$, that is, 


$$
F:[0,1]^{2} \rightarrow \Omega
$$

For each element $Q$ in the parametric domain $[0,1]^{2}$, there is a corresponding physical element $K=F(Q)$, as shown in Figure 1 .

We assume throughout that $F$ is invertible, with smooth inverse $F^{-1}$, on each element $Q \in Q_{h}$.

We define the physical mesh to be:

$$
\tau_{h}=\left\{K: K=F(Q), Q \in Q_{h}\right\}=F\left(Q_{h}\right) .
$$

We assume $\left(\tau_{h}\right)$ is quasi-uniform:

$$
\exists C>0, h \leq C h_{K} .
$$

with $h_{K}$ the diameter of $K$ and $h:=\max _{K \in \tau_{h}} h_{K}$.

We introduce $V_{h}$, the space spanned by B-splines basis functions in $\Omega$ as the push-forward of the B-splines space $S_{h}$.

$$
V_{h}:=\operatorname{span}\left\{N_{(i, j),\left(p_{1}, p_{2}\right)} \circ F^{-1}\right\}_{i=1, j=1}^{n_{1}, n_{2}} .
$$

Given a function $\hat{v} \in L^{2}(\hat{\Omega})$, we define a projective operator over the B-splines space $S_{h}$ as:

$$
\pi_{S_{h}}: L^{2}(\hat{\Omega}) \rightarrow S_{h}, \pi_{S_{h}} \hat{v}:=\sum_{i=1, j=1}^{n_{1}, n_{2}} \varphi(\hat{v}) N_{(i, j),\left(p_{1}, p_{2}\right)},
$$

where the linear functionals $\varphi \in L^{2}(\hat{\Omega})^{\prime}$ determine the dual basis for the set of B-splines.

The projective operator over the B-splines space $V_{h}$, is defined as the push-forward of the operator $\pi_{S_{h}}$.

$$
\pi_{h}: L^{2}(\Omega) \rightarrow V_{h}, \pi_{h} v:=\left(\pi_{S_{h}}(\hat{v})\right) \circ F^{-1}
$$

\section{Main Results}

This section is devoted to our convergence results obtained for respectively a polynomial of degree $k \geq 2$ and a polynomial of degree $k=1$. We present our main results whose proofs are given in the subsection 6.6.

Theorem 1. (Convergence for $R K 2, k \geq 2$ )

Assume the $\frac{4}{3}$ CFL Condition:
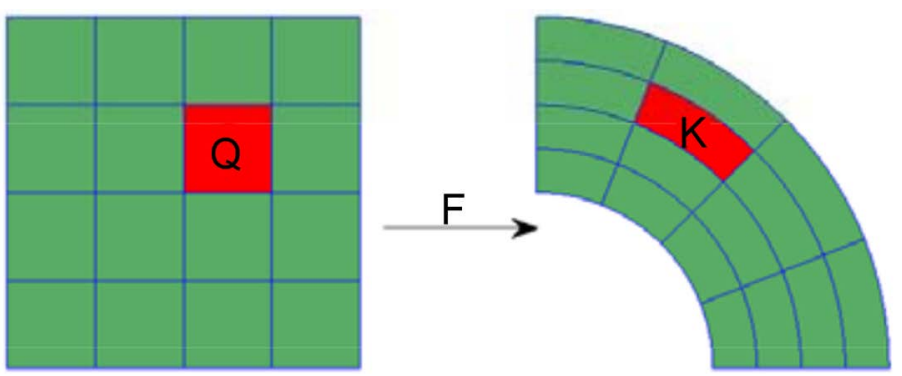

Figure 1. Definition of domains used in isogeometric analysis (Source [17]) (Page 181). 


$$
\delta t \leq \varrho^{\prime} \tau_{\star}^{-\frac{1}{3}}\left(\frac{h}{\beta_{c}}\right)^{\frac{4}{3}} \text { for some positive real number } \varrho^{\prime}
$$

and $d_{t}^{s} u \in C^{0}\left(H^{k+1-s}(\Omega)\right)$ for $s \in\{0,1\}$. Then,

$$
\left\|u^{N}-u_{h}^{N}\right\|_{L^{2}(\Omega)}+\left(\sum_{m=0}^{N-1} \delta t\left|u^{m}-u_{h}^{m}\right|_{\beta}^{2}\right)^{\frac{1}{2}} \lesssim \exp \left(\frac{4 C r^{8}}{\tau_{\star}} t_{f}\right)\left(\chi_{1} \delta t^{2}+\chi_{2} h^{k+\frac{1}{2}}\right)
$$

with

$$
\begin{gathered}
\chi_{1}=r^{4} t_{f}^{\frac{1}{2}} \tau_{\star}^{\frac{1}{2}}\left\|d_{t}^{3} u\right\|_{C^{0}\left(L^{2}(\Omega)\right)} \\
\chi_{2}=t_{f}^{\frac{1}{2}} r^{5} \beta_{c}^{\frac{1}{2}}\|u\|_{C^{0}\left(H^{k+1}(\Omega)\right)}+t_{f}^{\frac{1}{2}} r^{5} \beta_{c}^{-\frac{1}{2}}\left\|d_{t} u\right\|_{C^{0}\left(H^{k}(\Omega)\right)} \\
|v|_{\beta}^{2}=\int_{\partial \Omega} \frac{1}{2}|\beta \cdot n| v^{2}+\sum_{F \in \mathcal{F}_{h}^{i}} \int_{F} \frac{1}{2}\left|\beta \cdot n_{F}\right|[v]^{2},
\end{gathered}
$$

where

$$
r=\max \left(1, \sqrt{\max _{K \in \tau_{h}}\left(\lambda_{Q} \lambda_{K}\right)}\right)
$$

and $\delta t$ is the time step.

Theorem 2. (Convergence for $R K 2, k=1$ )

Assume the $\frac{4}{3}$ CFL Condition, assume $d_{t}^{s} u \in C^{0}\left(H^{k+1-s}(\Omega)\right)$ for $s \in\{0,1\}$ and $4-3 r^{2}>0$. Then,

$$
\begin{aligned}
& \left\|u^{N}-u_{h}^{N}\right\|_{L^{2}(\Omega)}+\left(\sum_{m=0}^{N-1} \delta t\left|u^{m}-u_{h}^{m}\right|_{\beta}^{2}\right)^{\frac{1}{2}} \\
& \lesssim \frac{1}{\sqrt{4-3 r^{2}}} \exp \left(\frac{8 C r^{8}}{\tau_{\star}} t_{f}\right)\left(\chi_{1} \delta t^{2}+\chi_{2} h^{k+\frac{1}{2}}\right)
\end{aligned}
$$

with

$$
\chi_{1}=r^{4} t_{f}^{\frac{1}{2}} \tau_{\star}^{\frac{1}{2}}\left\|d_{t}^{3} u\right\|_{C^{0}\left(L^{2}(\Omega)\right)}
$$

and

$$
\chi_{2}=t_{f}^{\frac{1}{2}} r^{5} \beta_{c}^{\frac{1}{2}}\|u\|_{C^{0}\left(H^{k+1}(\Omega)\right)}+t_{f}^{\frac{1}{2}} r^{5} \beta_{c}^{-\frac{1}{2}}\left\|d_{t} u\right\|_{C^{0}\left(H^{k}(\Omega)\right)}
$$

\section{Inverse and Trace Inequalities}

In this section, we present isogeometric inverse and trace inequalities, useful tools to analyze partial differential equations.

Let $K \in \tau_{h}$ and $Q=F^{-1}(K)$.

Theorem 3. (see [11] [18])

$$
\forall h>0, \forall K \in \tau_{h} \text { and } \forall v_{h} \in \mathbb{P}_{2}^{k}\left(\tau_{h}\right),\left\|\nabla v_{h}\right\|_{\left.L^{2}(K)\right]^{2}} \leq C_{\star} h_{K}^{-1}\left\|v_{h}\right\|_{L^{2}(K)}
$$


where $C_{\star}$ depends on $k$ and on the parametrization $F$.

Theorem 4. (see [16])

$$
\forall v_{h} \in \mathbb{P}_{2}^{k}\left(\tau_{h}\right),\left\|v_{h}\right\|_{L^{2}(\partial K)}^{2} \leq C \lambda_{Q} \lambda_{K} h_{K}^{-1}\left\|v_{h}\right\|_{L^{2}(K)}^{2}
$$

where $C$ depends only on $p_{1}$ and $p_{2}, \lambda_{Q}$ is the local shape regularity constant of $Q$, and $\lambda_{K}$ is the shape regularity constant of $K$.

We set $p:=\min \left\{p_{1}, p_{2}\right\} \quad$ (see. [4] [19]).

Theorem 5. (see [20]) Given the integers 1 and s such that $0 \leq l \leq s \leq p+1$ and a function $u \in H^{s}(\Omega)$, then:

$$
\sum_{K \in \tau_{h}}\left|u-\pi_{h} u\right|_{H^{l}(K)}^{2} \leq C h^{2(s-l)}\|u\|_{H^{s}(\Omega)}^{2},
$$

where $\mathrm{C}$ is independent on $h$.

Theorem 6. Given the integer $s$ such that $0 \leq s \leq p+1$ and a function $u \in H^{s}(\Omega)$, then:

$$
\sum_{K \in \tau_{h}}\left\|u-\pi_{h} u\right\|_{L^{2}(\partial K)}^{2} \leq C \max _{K \in \tau_{h}}\left(\lambda_{Q} \lambda_{K}\right) h^{(2 s-1)}\|u\|_{H^{s}(\Omega)}^{2},
$$

where $C$ is independent on $h$.

Proof 1. Let $u \in H^{s}(\Omega)$. Using the inequality (23), we have.

$$
\sum_{K \in \tau_{h}}\left\|u-\pi_{h} u\right\|_{L^{2}(\partial K)}^{2} \leq C \sum_{K \in \tau_{h}} \lambda_{Q} \lambda_{K} h_{K}^{-1}\left\|u-\pi_{h} u\right\|_{L^{2}(K)}^{2},
$$

$\left(\tau_{h}\right)_{h}$ being quasi-uniform, $h \leq C h_{K}$.

$$
\begin{aligned}
h \leq C h_{K} & \Rightarrow C^{-1} h_{K}^{-1} \leq h^{-1} \\
& \Rightarrow h_{K}^{-1} \leq C h^{-1} \\
& \Rightarrow C h_{K}^{-1} \leq C^{\prime} h^{-1}
\end{aligned}
$$

So

$$
\sum_{K \in \tau_{h}}\left\|u-\pi_{h} u\right\|_{L^{2}(\partial K)}^{2} \leq C \max _{K \in \tau_{h}}\left(\lambda_{Q} \lambda_{K}\right) h^{-1} \sum_{K \in \tau_{h}}\left\|u-\pi_{h} u\right\|_{L^{2}(K)}^{2}
$$

Using the inequality (24), we have:

$$
\sum_{K \in \tau_{h}}\left\|u-\pi_{h} u\right\|_{L^{2}(K)}^{2} \leq C h^{2 s}\|u\|_{H^{s}(\Omega)}^{2}
$$

Thus, we get:

$$
\sum_{K \in \tau_{h}}\left\|u-\pi_{h} u\right\|_{L^{2}(\partial K)}^{2} \leq C \max _{K \in \tau_{h}}\left(\lambda_{Q} \lambda_{K}\right) h^{(2 s-1)}\|u\|_{H^{s}(\Omega)}^{2}
$$

\section{Functional Setting and Space Semidiscretization}

\subsection{Functional Setting}

In this part, we introduce some basic notations for space-time functions and important theorems.

Theorem 7. (see. [11]) $C^{l}(V)$ is a Banach space when equipped with the norm:

$$
\|\phi\|_{C^{l}(V)}=\max _{0 \leq m \leq l}\left\|d_{t}^{m} \phi\right\|_{C^{0}(V)}
$$


with

$$
\|\phi\|_{C^{0}(V)}=\max _{t_{0} \leq t \leq t_{f}}\|\phi(t)\|_{V}[11] \text { (page69) }
$$

We want to specify mathematically the meaning of the boundary condition 1 . Our aim is to give a meaning to such traces in the space. Thus, we need to investigate the trace on $\partial \Omega$ of functions in the space defined by:

$$
L^{2}(|\beta \cdot n|, \partial \Omega)=\left\{v \text { is defined on } \partial \Omega, \int_{\partial \Omega}|\beta \cdot n| v^{2}<\infty\right\}
$$

\subsection{Space Semidiscretization}

Considering $\left(\tau_{h}\right)$, we present following notations:

- Interfaces are collected in the set $\mathcal{F}_{h}^{i}$ and boundary faces are collected in the set $\mathcal{F}_{h}^{b}$. We set $\mathcal{F}_{h}:=\mathcal{F}_{h}^{b} \cup \mathcal{F}_{h}^{i} . \forall T \in \tau_{h}, \mathcal{F}_{T}:=\left\{F \in \mathcal{F}_{h}, F \subset \partial T\right\}$.

- $\forall F \in \mathcal{F}_{h}^{i}$, the mean of $v$ is denoted by $\{\{v\}\}$.

- The jump of $v$ is denoted by $[v]$.

- Assume $P_{\Omega}=\left\{\Omega_{i}\right\}_{1 \leq i \leq N_{\Omega}}$ is a partition of $\Omega$ such that, for the exact solution $u$,

$$
u \in V_{\star}=V \cap H^{\frac{1}{2}+\varepsilon}\left(P_{\Omega}\right), \varepsilon>0
$$

where

$$
V=\left\{u \in L^{2}(\Omega), \nabla \cdot(\beta u) \in L^{2}(\Omega)\right\}
$$

We set

$$
V_{\star h}:=V_{\star}+V_{h} \text { with } V_{h}=\mathbb{P}_{2}^{k}\left(\tau_{h}\right)=\left\{v \in L^{2}(\Omega) ; \forall T \in \tau_{h}, v_{/ T} \in \mathbb{P}_{2}^{k}(T)\right\}
$$

We define the discrete operator $A_{h}: V_{\star h} \rightarrow V_{h}$ such as $\forall\left(v, w_{h}\right) \in V_{\star h} \times V_{h}$,

$$
\begin{aligned}
\left(A_{h} v, w_{h}\right)_{L^{2}(\Omega)}= & \int_{\Omega} \nabla \cdot(\beta v) w_{h}+\int_{\partial \Omega}(\beta \cdot n)^{\ominus} v w_{h} \\
& -\sum_{F \in \mathcal{F}_{h}^{i}} \int_{F}\left(\beta n_{F}\right)[v]\left\{\left\{w_{h}\right\}\right\}+\sum_{F \in \mathcal{F}_{h}^{i}} \frac{1}{2} \int_{F}\left|\beta \cdot n_{F}\right|[v]\left[w_{h}\right]
\end{aligned}
$$

\subsection{Assumptions}

For all $v \in V_{\star h}$, set:

$$
\begin{gathered}
\|v\|_{\star \star}^{2}=\|v\|_{u w b, \star}^{2}+\beta c h\|\nabla v\|_{L^{2}(\Omega)}^{2} \\
\|v\|_{u w b, \star}^{2}=\|v\|_{u w b}^{2}+\sum_{T \in \tau_{h}} \beta_{c}\|v\|_{L^{2}(\partial T)}^{2} \\
\|v\|_{u w b}^{2}=\frac{1}{\tau_{c}}\|v\|_{L^{2}(\Omega)}^{2}+|v|_{\beta}^{2} \\
E_{h}^{n}=\left\|\varepsilon_{\pi}^{n}\right\|_{\star \star}+\left\|\zeta_{\pi}^{n}\right\|_{\star \star}+\tau_{\star}^{\frac{1}{2}}\left\|d_{t}^{3} u\right\|_{C^{0}\left(L^{2}(\Omega)\right)} \delta t^{2}+\tau_{\star}^{-\frac{1}{2}}\left\|\varepsilon_{h}^{n}\right\|_{L^{2}(\Omega)}
\end{gathered}
$$

We abbreviate as $a \lesssim b$ the inequality $a \leq C b$ with positive $\mathrm{C}$ independent of $\beta, h, \delta t$. The value of $C$ can change at each occurrence [11].

We now state some assumptions on the discrete operator $A_{h}$. The first one (41) is important to introduce the notion of numerical fluxes: 
1) For all $\left(v, w_{h}\right) \in V_{\star h} \times V_{h}$,

$$
\begin{aligned}
\left(A_{h} v, w_{h}\right)= & -\int_{\Omega}\left(\beta \cdot \nabla w_{h}\right) v+\int_{\partial \Omega}(\beta \cdot n)^{\oplus} v w_{h} \\
& +\sum_{F \in \mathcal{F}_{h}^{i}} \int_{F}\left(\beta \cdot n_{F}\right)\{\{v\}\}\left[w_{h}\right]+\sum_{F \in \mathcal{F}_{h}} \frac{1}{2} \int_{F}\left|\beta \cdot n_{F}\right|[v]\left[w_{h}\right]
\end{aligned}
$$

2) From equality (41), Cauchy-Schwarz inequality and inverse inequality (22), we can infer:

For all $\left(v, w_{h}\right) \in H^{1}(\Omega) \times V_{h}$,

$$
\left(A_{h}\left(v-\pi_{h} v\right), w_{h}\right)_{L^{2}(\Omega)} \lesssim\left\|v-\pi_{h} v\right\|_{u w b, \star}\left\|w_{h}\right\|_{l w b}
$$

3) The three next assumptions are useful to bound the operator $A_{h}$.

For all

$$
v \in V_{\star h},\left\|A_{h} v\right\|_{L^{2}(\Omega)} \lesssim r \beta_{c}^{\frac{1}{2}} h^{-\frac{1}{2}}\|v\|_{\star \star} \text { with } r=\max \left(1, \sqrt{\max _{K \in \tau_{h}}\left(\lambda_{Q} \lambda_{K}\right)}\right)
$$

For all

$$
v_{h} \in V_{h},\left\|v_{h}\right\|_{\star \star} \lesssim r \beta_{c}^{\frac{1}{2}} h^{-\frac{1}{2}}\left\|v_{h}\right\|_{L^{2}(\Omega)}
$$

For all

$$
v_{h} \in V_{h},\left\|A_{h} v\right\|_{L^{2}(\Omega)} \lesssim r^{2} \beta_{c} h^{-1}\left\|v_{h}\right\|_{L^{2}(\Omega)}
$$

4) The two next inequalities are bounds of $\delta t\left(\alpha_{h}^{n}, \varepsilon_{h}^{n}\right)_{L^{2}(\Omega)}-\frac{\delta t}{2}\left|\varepsilon_{h}^{n}\right|_{\beta}^{2}$ and

$$
\begin{aligned}
& \delta t\left(\beta_{h}^{n}, \zeta_{h}^{n}\right)_{L^{2}(\Omega)}-\frac{\delta t}{2}\left|\zeta_{h}^{n}\right|_{\beta}^{2} \\
& \delta t\left(\alpha_{h}^{n}, \varepsilon_{h}^{n}\right)_{L^{2}(\Omega)}-\frac{\delta t}{2}\left|\varepsilon_{h}^{n}\right|_{\beta}^{2} \lesssim \delta t\left(E_{h}^{n}\right)^{2} \\
& \delta t\left(\beta_{h}^{n}, \zeta_{h}^{n}\right)_{L^{2}(\Omega)}-\frac{\delta t}{2}\left|\zeta_{h}^{n}\right|_{\beta}^{2} \lesssim r^{4} \delta t\left(E_{h}^{n}\right)^{2}
\end{aligned}
$$

5) The two last inequalities are obtained thanks to CFL condition and isogeometric inverse and trace inequalities:

$$
\begin{gathered}
\left\|\varepsilon_{\pi}^{m}\right\|_{\star \star}^{2} \lesssim r^{2} \beta_{c} h^{2 k+1}\left\|u^{m}\right\|_{H^{k+1}(\Omega)}^{2} \\
\left\|\zeta_{\pi}^{m}\right\|_{\star \star}^{2} \lesssim r^{2} \beta_{c} h^{2 k+1}\left(\left\|u^{m}\right\|_{H^{k+1}(\Omega)}^{2}+\beta_{c}^{-2}\left\|d_{t} u^{m}\right\|_{H^{k}(\Omega)}^{2}\right)
\end{gathered}
$$

For the time discretization, we are interested in an explicit scheme: the two stage Heun scheme.

\section{The Explicit Two Stage Heun Scheme Analysis}

In this section, we want to tackle the convergence analysis of the two stage Heun scheme.

\subsection{The Explicit Two Stage Heun Scheme}

Let $\delta t$ be the time step such as $t_{f}=N \delta t$ where $N$ is an integer. For 
$n \in\{0, \cdots, N\}$, we define the discrete times $t^{n}:=n \delta t$ and $u^{n}=u\left(t^{n}\right)$. Assume $\delta t \leq \tau_{\star}$ with $\tau_{\star}=\min \left(t_{f}, \tau_{c}\right)$.

We consider the following explicit scheme:

$$
\left\{\begin{array}{l}
u_{h}^{n, 1}=u_{h}^{n}-\delta t A_{h} u_{h}^{n} \\
u_{h}^{n+1}=\frac{1}{2}\left(u_{h}^{n}+u_{h}^{n, 1}\right)-\frac{1}{2} \delta t A_{h} u_{h}^{n, 1} \\
\text { with } u_{h}^{0}=\pi_{h} u^{0}
\end{array}\right.
$$

\subsection{Error Equation}

This step is to identify the error equation governing the time evolution of $\varepsilon_{h}^{n}$ and $\zeta_{h}^{n}$.

We set

$$
\begin{aligned}
& \varepsilon_{h}^{n}=u_{h}^{n}-\pi_{h} u^{n} \\
& \varepsilon_{\pi}^{n}=u^{n}-\pi_{h} u^{n} \\
& \zeta_{h}^{n}=w_{h}^{n}-\pi_{h} w^{n} \\
& \zeta_{\pi}^{n}=w^{n}-\pi_{h} w^{n}
\end{aligned}
$$

with

$$
w=u+\delta t d_{t} u \quad[11]
$$

From (50) and (51), we have $u^{n}-u_{h}^{n}=\varepsilon_{\pi}^{n}-\varepsilon_{h}^{n}$.

From (52) and (53), we have $w^{n}-w_{h}^{n}=\zeta_{\pi}^{n}-\zeta_{h}^{n}$.

We get:

$$
\begin{gathered}
\zeta_{h}^{n}=\varepsilon_{h}^{n}-\delta t A_{h} \varepsilon_{h}^{n}+\delta t \alpha_{h}^{n} \\
\varepsilon_{h}^{n+1}=\frac{1}{2}\left(\varepsilon_{h}^{n}+\zeta_{h}^{n}\right)-\frac{1}{2} \delta t A_{h} \zeta_{h}^{n}+\frac{1}{2} \delta t \beta_{h}^{n}
\end{gathered}
$$

where

$$
\alpha_{h}^{n}=A_{h} \varepsilon_{\pi}^{n}
$$

where

$$
\beta_{h}^{n}=A_{h} \zeta_{\pi}^{n}-\pi_{h} \theta^{n}
$$

and

$$
\theta^{n}=\frac{1}{\delta t} \int_{t^{n}}^{t^{n+1}}\left(t^{n+1}-t\right)^{2} u_{t}^{3} \mathrm{~d} t
$$

\subsection{Energy Identity}

This step is to derive an energy identity for our scheme (6.1).

$$
\begin{aligned}
& \left\|\varepsilon_{h}^{n+1}\right\|_{L^{2}(\Omega)}^{2}-\left\|\varepsilon_{h}^{n}\right\|_{L^{2}(\Omega)}^{2}+\delta t\left|\varepsilon_{h}^{n}\right|_{\beta}^{2}+\delta t\left|\zeta_{h}^{n}\right|_{\beta}^{2} \\
& =\left\|\varepsilon_{h}^{n+1}-\zeta_{h}^{n}\right\|_{L^{2}(\Omega)}^{2}+\delta t\left(\alpha_{h}^{n}, \varepsilon_{h}^{n}\right)_{L^{2}(\Omega)}+\delta t\left(\beta_{h}^{n}, \zeta_{h}^{n}\right)_{L^{2}(\Omega)}-\Lambda_{h}^{n}
\end{aligned}
$$

with 


$$
\Lambda_{h}^{n}=\delta t\left(\Lambda \varepsilon_{h}^{n}, \varepsilon_{h}^{n}\right)_{L^{2}(\Omega)}+\delta t\left(\Lambda \zeta_{h}^{n}, \zeta_{h}^{n}\right)_{L^{2}(\Omega)}
$$

\subsection{Stability Estimate}

Our aim is to bound the right terms in the energy identity (60).

We want now to establish a stability lemma for a polynomial of degree $k=1$ (68). To get it, we need the next lemma.

Lemma 1. Let $\pi_{h}^{0}$ denote the $L^{2}$-orthogonal projection onto $\mathbb{P}_{2}^{0}\left(\tau_{h}\right)$. $\mathbb{P}_{2}^{0}\left(\tau_{h}\right)$ is spanned by piecewise constant functions on $\tau_{h}$.

Then, $\forall\left(v_{h}, w_{h}\right) \in V_{h} \times V_{h}$,

$$
\left(A_{h} v_{h}, w_{h}-\pi_{h}^{0} w_{h}\right) \leq C_{\star \star} r \beta_{c}^{\frac{1}{2}} h^{-\frac{1}{2}}\left\|v_{h}\right\|_{u w b}\left\|w_{h}-\pi_{h}^{0} w_{h}\right\|_{L^{2}(\Omega)}
$$

where $C_{\star \star}$ is independent of $h, \delta t$ and of $\beta$.

Proof 2. This result is obtained using Cauchy-Schwarz inequality and equality (41).

\subsection{Preliminary Results}

This lemma is a preliminary stability bound.

Lemma 2. Assume $u \in C^{3}\left(L^{2}(\Omega)\right) \cap C^{0}\left(H^{1}(\Omega)\right)$.

Assume the CFL condition:

$$
\delta t \leq \varrho \frac{h}{\beta_{c}} \text { for some positive real number } \varrho .
$$

Thus,

$$
\left\|\varepsilon_{h}^{n+1}\right\|_{L^{2}(\Omega)}^{2}-\left\|\varepsilon_{h}^{n}\right\|_{L^{2}(\Omega)}^{2}+\frac{\delta t}{2}\left|\varepsilon_{h}^{n}\right|_{\beta}^{2}+\frac{\delta t}{2}\left|\zeta_{h}^{n}\right|_{\beta}^{2} \leq\left\|\varepsilon_{h}^{n+1}-\zeta_{h}^{n}\right\|_{L^{2}(\Omega)}^{2}+C r^{4} \delta t\left(E_{h}^{n}\right)^{2}
$$

where $C$ is independent of $h, \delta t$ and $\beta$.

Proof 3. Using CFL condition, energy identity (60), inequalities (46) and (47), we get (64).

Lemma 3. (Stability lemma, $k \geq 2)$ Assume $u \in C^{3}\left(L^{2}(\Omega)\right) \cap C^{0}\left(H^{1}(\Omega)\right)$. Assume the $\left(\frac{4}{3}\right.$ CFL Condition $)$

$$
\delta t \leq \varrho^{\prime} \tau_{\star}^{-\frac{1}{3}}\left(\frac{h}{\beta_{c}}\right)^{\frac{4}{3}} \text { for some positive real number } \varrho^{\prime}
$$

Then, we infer:

$$
\left\|\varepsilon_{h}^{n+1}\right\|_{L^{2}(\Omega)}^{2}-\left\|\varepsilon_{h}^{n}\right\|_{L^{2}(\Omega)}^{2}+\frac{\delta t}{2}\left|\varepsilon_{h}^{n}\right|_{\beta}^{2}+\frac{\delta t}{2}\left|\zeta_{h}^{n}\right|_{\beta}^{2} \leq C r^{8} \delta t\left(E_{h}^{n}\right)^{2}
$$

Proof 4. The stability lemma (66) for a polynomial of degree $k \geq 2$ is $o b$ tained by bounding the term $\left\|\varepsilon_{h}^{n+1}-\zeta_{h}^{n}\right\|_{L^{2}(\Omega)}^{2}$ in the energy identity (60).

Lemma 4. (Stability lemma, $k=1)$ Assume $u \in C^{3}\left(L^{2}(\Omega)\right) \cap C^{0}\left(H^{1}(\Omega)\right)$. Assume the CFL condition: 


$$
\begin{aligned}
& \delta t \leq \varrho \frac{h}{\beta_{c}} \text { for some positive real number } \varrho \\
& \text { with } \varrho \leq \min \left\{\frac{1}{8}\left(C^{\prime}\right)^{-2} ; \frac{1}{2}\left(C_{\star} C_{\star \star}\right)^{-\frac{2}{3}}\right\}
\end{aligned}
$$

Thus,

$$
\begin{aligned}
& \left\|\varepsilon_{h}^{n+1}\right\|_{L^{2}(\Omega)}^{2}-\left\|\varepsilon_{h}^{n}\right\|_{L^{2}(\Omega)}^{2}+\delta t\left(\frac{1}{2}-\frac{3}{8} r^{2}\right)\left|\varepsilon_{h}^{n}\right|_{\beta}^{2}+\delta t\left(\frac{1}{2}-\frac{3}{8} r^{2}\right)\left|\zeta_{h}^{n}\right|_{\beta}^{2} \\
& \leq C r^{4} \delta t\left(E_{h}^{n}\right)^{2}
\end{aligned}
$$

where $C$ is independent of $h, \delta t$ and $\beta$.

Proof 5. This lemma is proven as in [11] (Page 96).

\subsection{Proofs of Our Main Results}

\section{Proof of theorem 1}

$$
\begin{aligned}
&\left\|u^{N}-u_{h}^{N}\right\|_{L^{2}(\Omega)}=\left\|u^{N}-\pi_{h} u^{n}+\pi_{h} u^{n}-u_{h}^{N}\right\|_{L^{2}(\Omega)} \\
& \leq\left\|u^{N}-\pi_{h} u^{n}\right\|_{L^{2}(\Omega)}+\left\|\pi_{h} u^{n}-u_{h}^{N}\right\|_{L^{2}(\Omega)} \\
&\left\|u^{N}-u_{h}^{N}\right\|_{L^{2}(\Omega)} \leq\left\|\varepsilon_{\pi}^{N}\right\|_{L^{2}(\Omega)}+\left\|\varepsilon_{h}^{N}\right\|_{L^{2}(\Omega)}
\end{aligned}
$$

Using the triangle and Young inequalities, we deduce:

$$
\left(\sum_{m=0}^{N-1} \delta t\left|u^{m}-u_{h}^{m}\right|_{\beta}^{2}\right)^{\frac{1}{2}} \lesssim \sum_{m=0}^{N-1} \delta t^{\frac{1}{2}}\left\|\varepsilon_{\pi}^{m}\right\|_{\star \star}+\sum_{m=0}^{N-1} \delta t^{\frac{1}{2}}\left|\varepsilon_{h}^{m}\right|_{\beta}
$$

Thus, we obtain:

$$
\begin{aligned}
& \left\|u^{N}-u_{h}^{N}\right\|_{L^{2}(\Omega)}+\left(\sum_{m=0}^{N-1} \delta t\left|u^{m}-u_{h}^{m}\right|_{\beta}^{2}\right)^{\frac{1}{2}} \\
& \lesssim\left\|\varepsilon_{\pi}^{N}\right\|_{L^{2}(\Omega)}+\left\|\varepsilon_{h}^{N}\right\|_{L^{2}(\Omega)}+\sum_{m=0}^{N-1} \delta t^{\frac{1}{2}}\left\|\varepsilon_{\pi}^{m}\right\|_{\star \star}+\sum_{m=0}^{N-1} \delta t^{\frac{1}{2}}\left|\varepsilon_{h}^{m}\right|_{\beta}
\end{aligned}
$$

Let $n \in\{0, \cdots, N\}$

$$
\begin{gathered}
\text { Set } b^{n}=\frac{\delta t}{2}\left|\varepsilon_{h}^{n}\right|_{\beta}^{2}+\frac{\delta t}{2}\left|\zeta_{h}^{n}\right|_{\beta}^{2} \\
\text { Given }(a+b+c+d)^{2} \leq 4 a^{2}+4 b^{2}+4 c^{2}+4 d^{2}
\end{gathered}
$$

From the relation (66), we deduce that:

$$
\begin{array}{r}
\left\|\varepsilon_{h}^{n+1}\right\|_{L^{2}(\Omega)}^{2}+b^{n} \leq\left\|\varepsilon_{h}^{n}\right\|_{L^{2}(\Omega)}^{2}+C r^{8} \delta t\left(4\left\|\varepsilon_{\pi}^{n}\right\|_{\star \star}^{2}+4\left\|\zeta_{\pi}^{n}\right\|_{\star \star}^{2}\right. \\
\left.+4 \tau_{\star}\left\|d_{t}^{3} u\right\|_{C^{0}\left(L^{2}(\Omega)\right)}^{2} \delta t^{4}+4 \delta t \tau_{\star}^{-1}\left\|\varepsilon_{h}^{n}\right\|_{L^{2}(\Omega)}^{2}\right) \\
\text { Set } d^{n}=4 C r^{8} \delta t\left(\left\|\varepsilon_{\pi}^{n}\right\|_{\star \star}^{2}+\left\|\zeta_{\pi}^{n}\right\|_{\star \star}^{2}+\tau_{\star}\left\|d_{t}^{3} u\right\|_{C^{0}\left(L^{2}(\Omega)\right)}^{2} \delta t^{4}\right)
\end{array}
$$

whence, we have:

$$
\left\|\varepsilon_{h}^{n+1}\right\|_{L^{2}(\Omega)}^{2} \leq\left(1+4 C r^{8} \delta t \tau_{\star}^{-1}\right)\left\|\varepsilon_{h}^{n}\right\|_{L^{2}(\Omega)}^{2}+d^{n}-b^{n}
$$


Applying the Gronwall lemma, we get for $n=N-1$ :

$$
\left\|\varepsilon_{h}^{N}\right\|_{L^{2}(\Omega)}^{2} \leq \exp \left(\frac{4 C r^{8}}{\tau_{\star}}\left(t_{f}-t_{0}\right)\right)\left\|\varepsilon_{h}^{0}\right\|_{L^{2}(\Omega)}^{2}+\sum_{i=0}^{N-1} \exp \left(\frac{4 C r^{8}}{\tau_{\star}}\left(t_{f}-t_{i+1}\right)\right)\left(d^{i}-b^{i}\right)
$$

So

$$
\left\|\varepsilon_{h}^{N}\right\|_{L^{2}(\Omega)}^{2} \leq \sum_{i=0}^{N-1} \exp \left(\frac{4 C r^{8}}{\tau_{\star}}\left(t_{f}-t_{i+1}\right)\right)\left(d^{i}-b^{i}\right) \text { for } u_{h}^{0}=\pi_{h} u^{0} \Rightarrow \varepsilon_{h}^{0}=0
$$

So

$$
\left\|\varepsilon_{h}^{N}\right\|_{L^{2}(\Omega)}+\left(\sum_{i=0}^{N-1} \delta t^{\frac{1}{2}}\left|\varepsilon_{h}^{i}\right|_{\beta}\right) \lesssim\left(\sum_{i=0}^{N-1} \exp \left(\frac{4 C r^{8}}{\tau_{\star}} t_{f}\right) d^{i}\right)^{\frac{1}{2}}
$$

where

$$
d^{i}=4 C r^{8} \delta t\left(\left\|\varepsilon_{\pi}^{i}\right\|_{\star \star}^{2}+\left\|\zeta_{\pi}^{i}\right\|_{\star \star}^{2}+\tau_{\star}\left\|d_{t}^{3} u\right\|_{C^{0}\left(L^{2}(\Omega)\right)}^{2} \delta t^{4}\right)
$$

Therefore, we have:

$$
\left(\sum_{i=0}^{N-1} \delta t^{\frac{1}{2}}\left\|\varepsilon_{\pi}^{i}\right\|_{\star \star}\right) \lesssim\left(\sum_{i=0}^{N-1} \exp \left(\frac{4 C r^{8}}{\tau_{\star}} t_{f}\right) d^{i}\right)^{\frac{1}{2}}
$$

From inequalities (69), (74) and (75), we get:

$$
\left\|u^{N}-u_{h}^{N}\right\|_{L^{2}(\Omega)}+\left(\sum_{m=0}^{N-1} \delta t\left|u^{m}-u_{h}^{m}\right|_{\beta}^{2}\right)^{\frac{1}{2}} \lesssim\left\|\varepsilon_{\pi}^{N}\right\|_{L^{2}(\Omega)}+\exp \left(\frac{4 C r^{8}}{\tau_{\star}} t_{f}\right)\left(\sum_{i=0}^{N-1} d^{i}\right)^{\frac{1}{2}}
$$

From inequalities (48), (49) and CFL condition, we obtain:

$$
\begin{gathered}
\left(\sum_{i=0}^{N-1} d^{i}\right)^{\frac{1}{2}} \lesssim \\
t_{f}^{\frac{1}{2}} r^{5} \beta_{c}^{\frac{1}{2}} h^{k+\frac{1}{2}}\left\|u^{m}\right\|_{H^{k+1}(\Omega)}+t_{f}^{\frac{1}{2}} r^{5} \beta_{c}^{-\frac{1}{2}} h^{k+\frac{1}{2}}\left\|d_{t} u^{m}\right\|_{H^{k}(\Omega)} \\
+r^{4} t_{f}^{\frac{1}{2}} \tau_{\star}^{\frac{1}{2}}\left\|d_{t}^{3} u\right\|_{C^{0}\left(L^{2}(\Omega)\right)} \delta t^{2}
\end{gathered}
$$

Using inverse inequality (24),

$$
\left\|\varepsilon_{\pi}^{N}\right\|_{L^{2}(\Omega)}^{2} \lesssim h^{2 k+2}\left\|u^{m}\right\|_{H^{k+1}(\Omega)}^{2}
$$

So

$$
\left\|\varepsilon_{\pi}^{N}\right\|_{L^{2}(\Omega)} \lesssim h^{k+1}\|u\|_{C^{0}\left(H^{k+1}(\Omega)\right)}
$$

Therefore, inequality (76) becomes:

$$
\begin{aligned}
& \left\|u^{N}-u_{h}^{N}\right\|_{L^{2}(\Omega)}+\left(\sum_{m=0}^{N-1} \delta t\left|u^{m}-u_{h}^{m}\right|_{\beta}^{2}\right)^{\frac{1}{2}} \\
& \lesssim h^{k+1}\|u\|_{C^{0}\left(H^{k+1}(\Omega)\right)}+\exp \left(\frac{4 C r^{8}}{\tau_{\star}} t_{f}\right)\left(t_{f}^{\frac{1}{2}} r^{5} \beta_{c}^{\frac{1}{2}} h^{k+\frac{1}{2}}\|u\|_{C^{0}\left(H^{k+1}(\Omega)\right)}\right. \\
& \left.\quad+t_{f}^{\frac{1}{2}} r^{5} \beta_{c}^{-\frac{1}{2}} h^{k+\frac{1}{2}}\left\|d_{t} u\right\|_{C^{0}\left(H^{k}(\Omega)\right)}\right)+\exp \left(\frac{4 C r^{8}}{\tau_{\star}} t_{f}\right) r^{4} t_{f}^{\frac{1}{2}} \tau_{\star}^{\frac{1}{2}}\left\|d_{t}^{3} u\right\|_{C^{0}\left(L^{2}(\Omega)\right)} \delta t^{2}
\end{aligned}
$$

Set 


$$
\chi_{1}=r^{4} t_{f}^{\frac{1}{2}} \tau_{\star}^{\frac{1}{2}}\left\|d_{t}^{3} u\right\|_{C^{0}\left(L^{2}(\Omega)\right)}
$$

and

$$
\begin{gathered}
\chi_{2}=t_{f}^{\frac{1}{2}} r^{5} \beta_{c}^{\frac{1}{2}}\|u\|_{C^{0}\left(H^{k+1}(\Omega)\right)}+t_{f}^{\frac{1}{2}} r^{5} \beta_{c}^{-\frac{1}{2}}\left\|d_{t} u\right\|_{C^{0}\left(H^{k}(\Omega)\right)} \\
h^{k+1}\|u\|_{C^{0}\left(H^{k+1}(\Omega)\right)}=\sqrt{h} h^{k+\frac{1}{2}}\|u\|_{C^{0}\left(H^{k+1}(\Omega)\right)} \\
\lesssim t_{f}^{\frac{1}{2}} \beta_{c}^{\frac{1}{2}} h^{k+\frac{1}{2}}\|u\|_{C^{0}\left(H^{k+1}(\Omega)\right)} \text { for } h \lesssim t_{f} \beta_{c} \\
h^{k+1}\|u\|_{C^{0}\left(H^{k+1}(\Omega)\right)} \lesssim \chi_{2} h^{k+\frac{1}{2}}
\end{gathered}
$$

We obtain thus:

$$
\left\|u^{N}-u_{h}^{N}\right\|_{L^{2}(\Omega)}+\left(\sum_{m=0}^{N-1} \delta t\left|u^{m}-u_{h}^{m}\right|_{\beta}^{2}\right)^{\frac{1}{2}} \lesssim \exp \left(\frac{4 C r^{8}}{\tau_{\star}} t_{f}\right)\left(\chi_{1} \delta t^{2}+\chi_{2} h^{k+\frac{1}{2}}\right)
$$

Proof of theorem 2

Using inequality (68), we get inequality:

$$
\left\|\varepsilon_{h}^{N}\right\|_{L^{2}(\Omega)}^{2}+\sum_{i=0}^{N-1} b^{i} \leq \sum_{i=0}^{N-1} \exp \left(\frac{8 C r^{8}}{\tau_{\star}} t_{f}\right) d^{i}
$$

with

$$
\begin{gathered}
b^{i}=\delta t\left(\frac{1}{2}-\frac{3}{8} r^{2}\right)\left|\varepsilon_{h}^{i}\right|_{\beta}^{2}+\delta t\left(\frac{1}{2}-\frac{3}{8} r^{2}\right)\left|\zeta_{h}^{i}\right|_{\beta}^{2} \\
b^{i}=\delta t\left(\frac{4-3 r^{2}}{2}\right)\left|\varepsilon_{h}^{i}\right|_{\beta}^{2}+\delta t\left(\frac{4-3 r^{2}}{8}\right)\left|\zeta_{h}^{i}\right|_{\beta}^{2} \\
\left(\|\left.\varepsilon_{h}^{N}\right|_{L^{2}(\Omega)}+\sum_{i=0}^{N-1} \frac{\sqrt{4-3 r^{2}}}{2 \sqrt{2}} \delta t^{\frac{1}{2}}\left|\varepsilon_{h}^{i}\right|_{\beta}+\sum_{i=0}^{N-1} \frac{\sqrt{4-3 r^{2}}}{2 \sqrt{2}} \delta t^{\frac{1}{2}}\left|\zeta_{h}^{i}\right|_{\beta}\right)^{2} \\
\lesssim\left\|\varepsilon_{h}^{N}\right\|_{L^{2}(\Omega)}^{2}+\sum_{i=0}^{N-1} \delta t \frac{4-3 r^{2}}{8}\left|\varepsilon_{h}^{i}\right|_{\beta}^{2}+\sum_{i=0}^{N-1} \delta t \frac{4-3 r^{2}}{8}\left|\zeta_{h}^{i}\right|_{\beta}^{2}
\end{gathered}
$$

Thus

$$
\begin{aligned}
& \left\|\varepsilon_{h}^{N}\right\|_{L^{2}(\Omega)}+\sum_{i=0}^{N-1} \frac{\sqrt{4-3 r^{2}}}{2 \sqrt{2}} \delta t^{\frac{1}{2}}\left|\varepsilon_{h}^{i}\right|_{\beta}+\sum_{i=0}^{N-1} \frac{\sqrt{4-3 r^{2}}}{2 \sqrt{2}} \delta t^{\frac{1}{2}}\left|\zeta_{h}^{i}\right|_{\beta} \\
& \lesssim\left(\sum_{i=0}^{N-1} \exp \left(\frac{8 C r^{8}}{\tau_{\star}} t_{f}\right) d^{i}\right)^{\frac{1}{2}}
\end{aligned}
$$

So

$$
\left\|\varepsilon_{h}^{N}\right\|_{L^{2}(\Omega)}+\sum_{i=0}^{N-1} \sqrt{4-3 r^{2}} \delta t^{\frac{1}{2}}\left|\varepsilon_{h}^{i}\right|_{\beta} \lesssim\left(\sum_{i=0}^{N-1} \exp \left(\frac{8 C r^{8}}{\tau_{\star}} t_{f}\right) d^{i}\right)^{\frac{1}{2}}
$$

whence

$$
\left\|\varepsilon_{h}^{N}\right\|_{L^{2}(\Omega)}+\sum_{i=0}^{N-1} \delta t^{\frac{1}{2}}\left|\varepsilon_{h}^{i}\right|_{\beta} \lesssim \frac{1}{\sqrt{4-3 r^{2}}}\left(\sum_{i=0}^{N-1} \exp \left(\frac{8 C r^{8}}{\tau_{\star}} t_{f}\right) d^{i}\right)^{\frac{1}{2}} \text { for } \sqrt{4-3 r^{2}} \leq 1
$$


Therefore,

$$
\begin{aligned}
& \left\|u^{N}-u_{h}^{N}\right\|_{L^{2}(\Omega)}+\left(\sum_{m=0}^{N-1} \delta t\left|u^{m}-u_{h}^{m}\right|_{\beta}^{2}\right)^{\frac{1}{2}} \\
& \lesssim h^{k+1}\|u\|_{C^{0}\left(H^{k+1}(\Omega)\right)}+\frac{1}{\sqrt{4-3 r^{2}}} \exp \left(\frac{8 C r^{8}}{\tau_{\star}} t_{f}\right) \\
& \times\left(t_{f}^{\frac{1}{2}} r^{5} \beta_{c}^{\frac{1}{2}} h^{k+\frac{1}{2}}\|u\|_{C^{0}\left(H^{k+1}(\Omega)\right)}+t_{f}^{\frac{1}{2}} r^{5} \beta_{c}^{-\frac{1}{2}} h^{k+\frac{1}{2}}\left\|d_{t} u\right\|_{C^{0}\left(H^{k}(\Omega)\right)}\right) \\
& +\frac{1}{\sqrt{4-3 r^{2}}} \exp \left(\frac{8 C r^{8}}{\tau_{\star}} t_{f}\right) r^{4} t_{f}^{\frac{1}{2}} \tau_{\star}^{\frac{1}{2}}\left\|d_{t}^{3} u\right\|_{C^{0}\left(L^{2}(\Omega)\right)} \delta t^{2} \\
& \left\|u^{N}-u_{h}^{N}\right\|_{L^{2}(\Omega)}+\left(\sum_{m=0}^{N-1} \delta t\left|u^{m}-u_{h}^{m}\right|_{\beta}^{2}\right)^{\frac{1}{2}} \\
& \lesssim \frac{1}{\sqrt{4-3 r^{2}}} h^{k+1}\|u\|_{C^{0}\left(H^{k+1}(\Omega)\right)}+\frac{1}{\sqrt{4-3 r^{2}}} \exp \left(\frac{8 C r^{8}}{\tau_{\star}} t_{f}\right) \\
& \times\left(t_{f}^{\frac{1}{2}} r^{5} \beta_{c}^{\frac{1}{2}} h^{k+\frac{1}{2}}\|u\|_{C^{0}\left(H^{k+1}(\Omega)\right)}+t_{f}^{\frac{1}{2}} r^{5} \beta_{c}^{-\frac{1}{2}} h^{k+\frac{1}{2}}\left\|d_{t} u\right\|_{C^{0}\left(H^{k}(\Omega)\right)}\right) \\
& +\frac{1}{\sqrt{4-3 r^{2}}} \exp \left(\frac{8 C r^{8}}{\tau_{\star}} t_{f}\right) r^{4} t_{f}^{\frac{1}{2}} \tau_{\star}^{\frac{1}{2}}\left\|d_{t}^{3} u\right\|_{C^{0}\left(L^{2}(\Omega)\right)} \delta t^{2} \text { for } 1 \leq \frac{1}{\sqrt{4-3 r^{2}}} \\
& \left\|u^{N}-u_{h}^{N}\right\|_{L^{2}(\Omega)}+\left(\sum_{m=0}^{N-1} \delta t\left|u^{m}-u_{h}^{m}\right|_{\beta}^{2}\right)^{\frac{1}{2}} \\
& \lesssim \frac{1}{\sqrt{4-3 r^{2}}} \exp \left(\frac{8 C r^{8}}{\tau_{\star}} t_{f}\right)\left(\chi_{1} \delta t^{2}+\chi_{2} h^{k+\frac{1}{2}}\right)
\end{aligned}
$$

Remark 1. When $F$ is the identity mapping, $K=Q$ so $\lambda_{K}=\lambda_{Q}=1$ [16] (page 10). Therefore $r=1$. We get same results as Alexandre Ern. Thus, Our results are a generalization of Ern results because in finite elements method, Ern obtained his error estimate, working on a polygon [11]. In the framework of our work, we got the same order of precision in time and space like Alexandre Ern. But our result is obtained for anygeometry.

\section{Conclusion}

The isogeometric method has been used to establish an error estimate for transport equation in $2 \mathrm{D}$ using the explicit two stage Heun scheme, for smooth solutions, in the energy norm comprising the $L^{2}$-norm and the jumps. These results generalize Ern results. An extension of this present paper is to tackle Burgers equation to get an isogeometric error estimate.

\section{Acknowledgements}

This article has been written in the framework of my Phd works, supported by CEA-SMA (Centre d'Excellence Africain en Sciences Mathématiques et Applications), funded by World Bank. 


\section{Conflicts of Interest}

The authors declare that there is no conflict of interest as far as the publication of this paper is concerned.

\section{References}

[1] Hubbard, J. and Hubert, F. (2005) Calcul scientifique, de la théorie à la pratique. Université de Provence, Marseille.

[2] Salsa, S. (2008) Partial Differential Equations in Action, from Modelling to Theory. Département de mathématiques polytechniques de Milan, Milan.

[3] Cottrell, J.A., Hughes, T.J.R. and Bazilevs, Y. (2009) Isogeometric Analysis, toward Integration of CAD and FEA. John Wiley and Sons, Hoboken. https://doi.org/10.1002/9780470749081

[4] Bazilevs, Y., Beirao da Veiga, L., Cottrell, A., Hughes, T. and Sangalli, G. (2006) Isogeometric Analysis: Approximation, Stability and Error Estimates for H-Refined Meshes. University of Texas at Austin, Institute for Computational Engineering and Sciences, Dipartimento di Mathematica "F. Enriques", Universita di Milano, Dipartimento di Mathematica "F. Casorati”, University of Pavia, Pavia.

[5] Hughes, T.J.R., Reali, A. and Sangalli, G. (2008) Efficient Quadrature for NURBS Based Isogeometric Analysis. Elsevier, Amsterdam.

[6] Duvigneau, R. (2013) Conception optimale en mécanique des fluides numériques: Approches hiérarchiques, robustes et isogeometriques. Université de Nice Sophia Antipolis, Antipolis.

[7] Cockburn, B. and Shu, C.-W. (1989) TVB Runge-Kutta Local Projection Discontinuous Galerkin Finite Element Method for Conservation Laws II: General Framework. Mathematics of Computation, 52, 411-435.

[8] Cockburn, B., Lin, S. and Shu, C.-W. (1989) TVB Runge-Kutta Local Projection Discontinuous Galerkin Finite Element Method for Conservation Laws III: One-Dimensional Systems. Journal of Computational Physics, 84, 90-113. https://doi.org/10.1016/0021-9991(89)90183-6

[9] Cockburn, B., Hou, S. and Shu, C.-W. (1990) The Runge-Kutta Local Projection Discontinuous Galerkin Finite Element Method for Conservation Laws IV: The Multidimensional Case. Mathematics of Computation, 54, 545-581.

[10] Cockburn, B., Karniadakis, G.E. and Shu, C.-W. (2000) Discontinuous Galerkin Methods-Theory, Computation and Applications. Lecture Notes in Computational Science and Engineering 11, Springer-Verlag, New York. https://doi.org/10.1007/978-3-642-59721-3

[11] Di Pietro, D.A. and Ern, A. (2012) Mathematical Aspects of Discontinuous Galerkin Methods. Springer-Verlag, Berlin. https://doi.org/10.1007/978-3-642-22980-0

[12] Burman, E., Ern, A. and Fernandez, M.A. (2010) Explicit Runge-Kutta Schemes and Finite Elements with Symmetric Stabilization for First-Order Linear PDE Systems. Society for Industrial and Applied Mathematics, 48, 2019-2042. https://doi.org/10.1137/090757940

[13] Johannessen, K.A. (2009) An Adaptive Isogeometric Finite Element Analysis. Master of Science in Physics and Mathematics, Norwegian University of Science and Technology, Trondheim.

[14] Gahalaut, K.P.S. (2013) Isogeometric Analysis: Condition Number Estimates and Fast Solvers. PhD, Technisch-Naturwissenschaftliche Fakultät, Linz. 
[15] Farin, G. (1997) Curves and Surfaces for Computer Aided Geometric Design, a Practical Guide. Fourth Edition, Academic Press, Cambridge.

[16] Evans, J. and Hughes, T. (2011) Explicit Trace Inequalities for Isogeometric Analysis and Parametric Hexahedral Finite Elements. The Institute for Computational Engineering and Sciences Report, the University of Texas, Austin. https://doi.org/10.21236/ADA555335

[17] Beirao da Veiga, L., Buffa, A., Sangalli, G. and Vazquez, R. (2014) Mathematical Analysis of Variational Isogeometric Methods. Cambridge University Press, Cambridge. https://doi.org/10.1017/S096249291400004X

[18] Ern, A. and Guermond, J.L. (2004) Theory and Practice of Finite Elements. Springer-Verlag, Berlin.

[19] Schumacher, L. (2013) Isogeometric Analysis for Scalar Convection Diffusion Equations. PhD Thesis, Mathematisch-Naturwissenschaftlicke Fakultat II Institut fur Mathematik, Mai.

[20] Tagliabue, A., Dedè, L. and Quarteroni, A. (2012) Isogeometric Analysis and Error Estimates for High Order Partial Differential Equations in Fluid Dynamics. Mathematics Institute of Computational Science and Engineering Technical Report, October 2012. 